which proved prevalence are different depending on race (10.35\% Asian vs $1.51 \%$ White; $P=0.027$ ).

Conclusions We firstly report primary sites and racial heterogeneity of POLE/POLD1 mutation in CRC to call for more attention when designing clinical trials and data analysis.

\section{IDDF2020-ABS-0039 TO COMPARE THE TISSUE DIAGNOSTIC YIELD OF SOLID LESION BIOPSIES BASED ON THE HISTOPATHOLOGICAL ANALYSIS OF ENDOSCOPIC ULTRASOUND-GUIDED FINE NEEDLE ASPIRATION (EUS-FNA) SAMPLES PRODUCED BY THE 19G PROCORE NEEDLE, STANDARD 19G NEEDLE AND 22G PROCORE NEEDLE}

Mahesh Kumar Gupta*. Medanta-The Medicity, Gurgaon, Haryana, India

\subsection{6/gutjnl-2020-IDDF.48}

Background EUS is a sensitive method for detecting intestinal and extra-intestinal mass lesions including lymphadenopathy. FNA allows evaluating cellular findings suggestive of malignancy but inflammation causes cellular changes undistinguishable from neoplasia solely based on the cytological evaluation, because tissue architecture and cell morphology are essential for accurate pathological assessment. Various EUS-guided techniques have been explored to retrieve tissue specimens with variable success and complication rates.

Methods All the Patients, above 18 years of age, having intestinal and extra-intestinal solid mass lesions including lymphadenopathy, were subjected to EUS guided FNA. Patients with cystic lesions refused to sign the informed consent and with coagulopathy (INR $>1.5$, Platelets <50000) were excluded from the study.

Results Total 215 patients were evaluated, out of which EUS-FNA was technically feasible in 210 (97.67\%) cases. Three needle passes were made in every case. There was no significant difference between these three groups with regard to the age (p-value-0.676), gender ( $p$-value-0.856), location (p-value-0.998), echogenicity ( $p$-value-0.123), border (p-value-0.216), size (p-value-0.735 \& 0.374) of the lesions and presence of calcification ( $\mathrm{p}$-value-0.093)or necrosis (p-value 0.729). Sample suitable for pathological evaluation was obtained in $90.5 \%$ cases with a tissue core in $45.7 \%$ cases. $28.1 \%$ lesions were malignant, $62.4 \%$ were benign and $9.5 \%$ remained undiagnosed. The histopathological diagnoses were possible in $87.1 \%, 90.0 \%$ and $94.3 \%$ cases respectively with 22G Procore, 19G Procore and 19G Standard needles (p-value-.350). Samples for the presence of blood clot in order of $19 \mathrm{G}$ procore $(70.00 \%)>22 \mathrm{G}$ procore $(50.00 \%)>19$ G Standard (42. 8\%),(P-value 0.003). There were no post-procedure complications noted in any group.

Conclusions Procore needles did not offer the extra possibility of obtaining a core sample for histopathological analysis in this study, but there is a high possibility of the presence of blood clots. Any of these three needles can be used for biopsy according to the availability and expertise of the endosonologist. The outcome depends on the experience of the endosonologist as well as the pathologist.

\section{IDDF2020-ABS-0041 PANCREATIC NEUROENDOCRINE TUMORS: CORRELATION BETWEEN THE SONOGRAPHIC FEATURES AND THE PATHOLOGICAL TUMOR GRADE}

Jingzhi Huang*, Xiaohua Xie, Manxia Lin, Ming Xu, Guangliang Huang, Xiaoyan Xie. Department of Medical Ultrasound, Division of Interventional Ultrasound, The First Affiliated Hospital, Sun Yat-sen University, China

\subsection{6/gutjnl-2020-IDDF.49}

Background It is confirmed that patients' prognosis of pancreatic neuroendocrine tumors (pNETs) were different according to different pathological grades. Imagings may predict tumor grades. We intended to analyse the value of contrast-enhanced ultrasound (CEUS) in the pathological classification of pNETs. Methods Eighty-six pNETs patients who underwent CEUS before pathologic diagnosis were retrospectively reviewed. Ultrasonographic features and enhancement pattern in each phase were analyzed among the three pathologic grades of pNETs.

Results Eighty-six pNETs included 45 G1(52.3\%), 29 G2 (33.7\%), 12 G3 (14.0\%). 48.9\% (22/45) G1 tumors were less than $2 \mathrm{~cm}$, while $93.1 \%(27 / 29)$ G2 and $100 \%(12 / 12)$ G3 were larger than $2 \mathrm{~cm}$ (G1 vs. G2, $p<0.001$; G1 vs. G3, $p$ $=0.001) .58 .3 \%(7 / 12)$ G3 tumors had pancreatic duct dilatation and $41.7 \%(5 / 12)$ G3 had hepatic metastasis, which were more common than G1 tumors with both only 4.4\% (2/45) had pancreatic duct dilatation or hepatic metastasis $(p<$ 0.001, $p=0.002$ ). On CEUS, G1 tumors ((figure 1A,1B) showed homogeneously hyper-enhancement in the early phase) more often menifested hyper- or iso- enhancement in the

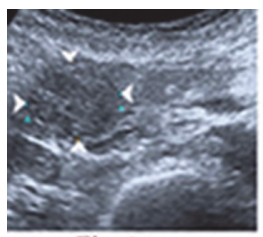

Fig. 1A

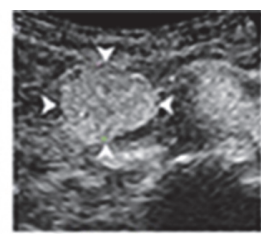

Fig. IB

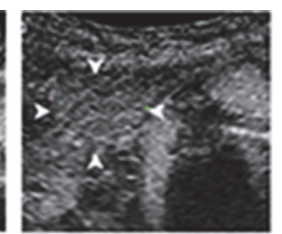

Fig. IC
Abstract IDDF2020-ABS-0041 Figure 1

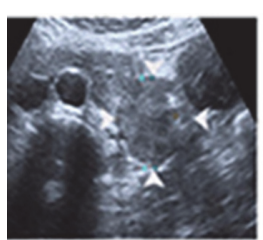

Fig. 2A

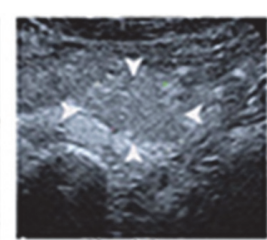

Fig. 2B

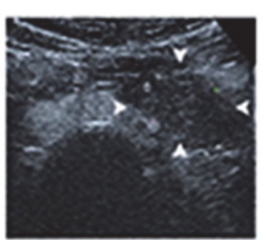

Fig. 2C
Abstract IDDF2020-ABS-0041 Figure 2

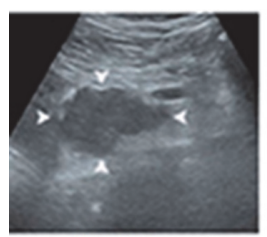

Fig. 3A

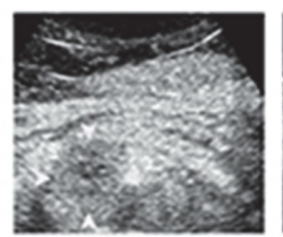

Fig. 3B

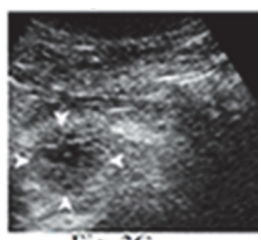

Fig. $3 \mathrm{C}$
Abstract IDDF2020-ABS-0041 Figure 3 
early phase (0-30s) than G2 ((figure 2A,2B) showed homogeneously iso-enhancement in the early phase) and G3 ((figure $3 \mathrm{~A}, 3 \mathrm{~B})$ showed inhomogeneously iso-enhancement in the early phase) tumors $(p=0.006, p<0.001)$. Besides, the incidence rate of hyper-enhancement in the early phase was higher in G2 tumors than G3 tumors $(p=0.009)$. While in the late phase (31-120s), the incidence rate of iso-enhancement in G1 tumor ((figure 1C) showed iso-enhancement in the late phase) was higher than G2 ((figure 2C) showed hypo-enhancement in the late phase) and G3 ((figure 3C) showed hypo-enhancement in the late phase $)$ tumors $(p=0.001, p<0.001)$. Heterogeneous enhancement and non-enhanced area appeared more often in G2 tumor than G1 tumor $(\mathrm{p}=0.007, \mathrm{p}=0.002)$.

Conclusions Sonographic features, such as tumor size, pancreatic duct dilatation or hepatic metastasis as well as enhancement pattern in each phase may help to predict different pathologic grade of pNETs.

\section{IDDF2020-ABS-0042 HYPERTROPHIC CECAL TUBERCULOSIS: A CASE REPORT}

Marc Ryan Pascua*. De La Salle University Medical Center, Philippines

10.1136/gutjnl-2020-IDDF.50

Background Gastrointestinal tuberculosis (GITB) remains to be a major health problem in developing countries and results in significant morbidity and mortality. It can present with diagnostic challenges, and a high index of suspicion should be considered specifically in endemic regions. Clinical, radiographic, endoscopic, and histopathologic findings complement one another in establishing the diagnosis. It may involve any part of the gastrointestinal tract. Isolated colonic tuberculosis is less common. The hypertrophic type resembles an inflammatory mass that mimics malignant neoplasm, which can cause intestinal obstruction. It is important to recognize GITB early to prevent complications and the possible need for surgery. We report a hypertrophic type of isolated cecal tuberculosis mimicking colonic malignancy.

Methods A 56y/o male with chronic renal insufficiency presented with intermittent, colicky abdominal pain associated

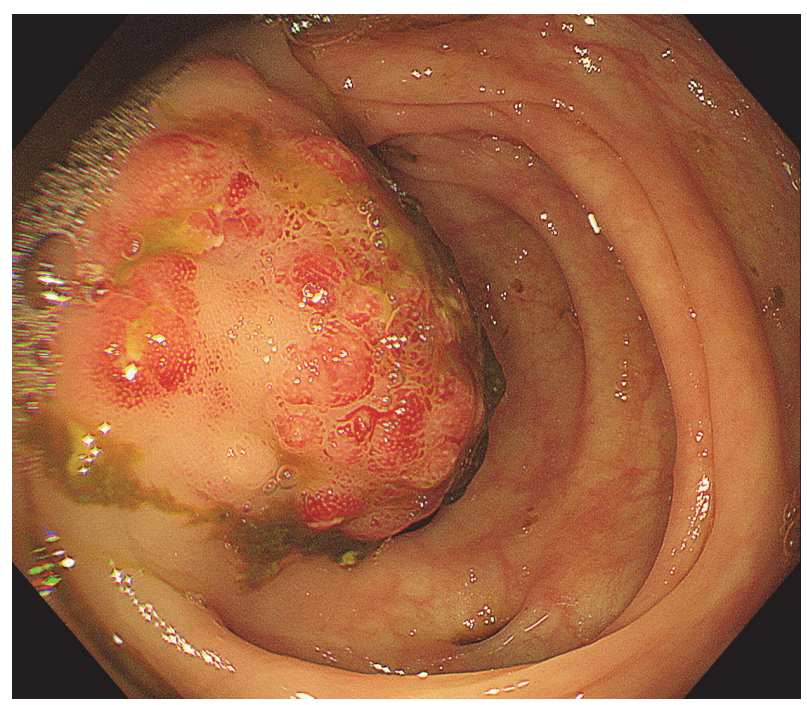

Abstract IDDF2020-ABS-0042 Figure 1 Cecal mass almost completely obstructing the lumen with changes in bowel movement and weight loss. He presented with fever, a slightly distended abdomen with hyperactive bowel sounds.

Results Complete blood count revealed anemia (Hgb 9.9 g/ $\mathrm{dL})$, leukocytosis $\left(17.3 \times 10^{9} / \mathrm{L}\right)$ and elevated serum creatinine (1166 umol/L). Abdominal CT scan showed ascending colon wall thickening with associated pericecal fat stranding and luminal narrowing. Colonoscopy showed a large mass at the cecum near the ileocecal valve.

Histopathology showed chronic granulomatous inflammation with Langhans giant cells and necrosis consistent with cecal tuberculosis.

Anti-TB treatment for category 1 extra-pulmonary TB consists of two (2) months of isoniazid, rifampicin, pyrazinamide, and ethambutol (2HRZE) as intensive phase followed by four (4) months of isoniazid and rifampicin (4HR) as continuation phase.

Conclusions Differential diagnosis of gastrointestinal TB should always be considered in colonic masses producing obstruction among patients in TB-endemic countries. (Figure 1).

\section{IDDF2020-ABS-0043 INTERVAL TIME BETWEEN GASTRIC PREPARATION AND MAGNETIC- CONTROLLED CAPSULE ENDOSCOPY: A RANDOMIZED CONTROLLED TRIAL}

Jia-chuan Wu*, Rui-yan Chen, Xiao-dong Chen, Li-fang Ye, Xiao-qiao Yang, Biao Liang, Hai-zhu Li, Li-li Ye. Guangdong Second Provincial General Hospital, China

\subsection{6/gutjnl-2020-IDDF.51}

Background To investigate the best interval time between gastric preparation and magnetic-controlled capsule endoscopy by comparing the effect of various interval time on image quality of stomach, safety, tolerance and positive lesions through a randomized controlled trial.

Methods 80 patients referred for MCE from Jan. 2018 to Dec. 2018 were randomly assigned to three groups, interval time with 30 minute and less (A), 30 to 60 minutes (B) or 60 minutes and more (C). All patients fasted for at least 8 hours before MCE and administrated with dimethicone, pronase and sodium bicarbonate. Cleanliness and visualization scores, safety, tolerability, additional water required and diagnostic sensitivity were compared. A multi-factor analysis of cleanliness and visualization scores was conducted.

Results The total cleanliness scores were (mean \pm SD) 20.44 \pm 2.61 (A), $20.96 \pm 1.68$ (B), and $20.56 \pm 2.90$ (C). The total visualization scores (mean $\pm \mathrm{SD}$ ) were $16.33 \pm 2.01$ (A), 16.41 \pm 1.50 (B), and $16.07 \pm 1.96$ (C). There was no statistical difference among groups $\mathrm{A}, \mathrm{B}$ and $\mathrm{C}(\mathrm{P}>0.05)$. MCE detected positive findings in $16(59.3 \%), 18(66.7 \%)$ and $15(55.6 \%)$ patients in group $\mathrm{A}, \mathrm{B}$ and $\mathrm{C}$ respectively, with no significant difference between groups $(\mathrm{P}>0.05)$. Additional water required during MCE were (mean $\pm \mathrm{SD})$ 107.41 $\pm 70.31 \mathrm{~mL}(\mathrm{~A}), 46.30$ $\pm 39.04 \mathrm{~mL}(\mathrm{~B}), 79.63 \pm 65.43 \mathrm{~mL}(\mathrm{C})$, and it was much less in group $\mathrm{B}$ than the other two groups $(\mathrm{P}=0.003)$. There was a negative correlation between cleanliness and visualization scores and the infection of $\mathrm{H}$. pylori $\left(\mathrm{r}_{\mathrm{s}}=-0.326, \mathrm{P}=0.003\right.$ and $\left.r_{s}=-0.235, P=0.035\right)$.

Conclusions Patients administrated with dimethicone, pronase and sodium bicarbonate 30 minute and less, 30 to 60 minutes, 60 minutes and more were not statistically different 